\title{
A teleparallel type theory for massless spin 2 fields
}

\author{
J. W. Maluf ${ }^{1}$ and S. C. Ulhoa ${ }^{2}$ \\ Instituto de Física, Universidade de Brasília \\ 70.919-970 Brasília DF, Brazil
}

October 19, 2020

\begin{abstract}
We present the Lagrangian and Hamiltonian formulations of a theory for spin 2 fields. The construction is developed in flat space-time. The construction in curved space-time is conceptually straightforward, although it is not unique. The theory is based on a symmetric tensor $S_{\mu \nu}$, contains two degrees of freedom of radiation, is motivated by the teleparallel formulation of general relativity, and displays a certain resemblance with Maxwell's theory for the electromagnetic field.
\end{abstract}

(1) jwmaluf@gmail.com,wadih@unb.br

(2) sc.ulhoa@gmail.com 


\section{Introduction}

It is generally accepted that a theory for spin 2 fields is based on second order symmetric tensors. However, there is no consensus as to what would be the theory for these symmetric tensors. One standard approach for obtaining field equations for massless spin 2 fields is the linearisation of Einstein's equations. A second standard approach, although not completely independent from the former, is given by the Fierz-Pauli theory $[1,2]$. In the case of massive spin 2 fields, there are also several investigations in the literature (see, for instance, Refs. [3, 4, 5] and references therein). Theories with slightly massive gravitons have attracted attention recently, as they could be relevant for the cosmological constant problem. Unlike the case of spin 1 fields described by Maxwell's theory, the interaction of spin 2 fields with other fields is not a trivial issue, as it is not entirely clear what is the source (or sources) of the massless spin 2 fields. Electric charges and currents are sources of the electromagnetic field, but a similar physical description is not known to exist for spin 2 fields. As we will discuss at the end of this article, the gravitational field might be one source for spin 2 fields.

In this article we present a new theory for the dynamics of spin 2 fields. The theory follows from the analysis developed in Ref. [6], and is based on a Lagrangian density similar to the one adopted in the teleparallel equivalent of general relativity (TEGR) [7,8], the difference being that instead of using tetrad fields, as is normally done in the TEGR, now one redefines the Lagrangian density in terms of a symmetric tensor $S_{\mu \nu}$. By adopting a suitable gauge condition, the symmetric tensor obeys the wave equation for massless fields. Although the structure of the theory in consideration here is very much different from the theory for spin 1 fields, it shares similarities with Maxwell's theory for the electromagnetic field.

In section 2 we present the Lagrangian formulation of the theory, although most of the content of this section was already presented in Ref. [6]. In section 3, the Hamiltonian formulation of the theory is developed. At the end of this section we show that the theory describes two degrees of freedom of radiation, in similarity to the degrees of freedom that arise in the linearisation of Einstein's equations, and that yield linearised gravitational waves. In section 4 we calculate the energy density of the spin 2 fields, assuming the same gauge that is used in the linearisation of gravitational waves. In the final section we discuss the interaction of the spin 2 fields with the gravitational field. We show that there is a natural minimal coupling with the gravitational 
field, but the coupling seems to be different if the background space-time geometry is either the Weitzenböck or Riemannian space.

\section{The Lagrangian formulation}

The geometrical framework of the theory considered in this article has already been presented in Ref. [6]. The motivation is to look for an extension of the standard teleparallel equivalent of general relativity (TEGR) by considering the distorted torsion tensor, a quantity that was introduced by Okubo [9]. The distorted torsion tensor is defined in terms of the tetrad fields and of a tensor $S_{\mu}^{\lambda}$ which, in turn, defines the Nijenhuis tensor [10, 11]. It was observed that in flat space-time limit of the models considered in Ref. [6], the symmetric form of this tensor obeys the usual wave equation, a feature that also holds in curved space-time.

In the present analysis, the focus is only on the tensor $S_{\lambda \mu}=S_{\mu \lambda}$, in order to investigate the essential features of the theory. For this purpose, we will treat the theory just like we address Maxwell's theory in the standard way. The theory considered below is formulated in flat space-time, and is invariant under Lorentz transformations. The introduction of a flat spacetime metric tensor, or flat space-time tetrad fields, to build a theory invariant under general coordinate transformations, will be carried out in the future, only after the relevant aspects of the theory are displayed and understood.

We will use, as much as possible, the same notation of Ref. [6]. The flat space-time metric tensor is $\eta_{\mu \nu}=(-1,+1,+1,+1)$, and $S_{\lambda \mu}=\eta_{\lambda \rho} S_{\mu}^{\rho}$, $S^{\rho \sigma}=S_{\mu}^{\rho} \eta^{\mu \sigma}$. We define the tensor

$$
\mathcal{T}_{\lambda \mu \nu}=\partial_{\mu} S_{\lambda \nu}-\partial_{\nu} S_{\lambda \mu}=-\mathcal{T}_{\lambda \nu \mu}
$$

The Lagrangian density for the theory of spin 2 fields is defined by

$$
L=-\frac{1}{2} k\left(\frac{1}{4} \mathcal{T}^{\lambda \mu \nu} \mathcal{T}_{\lambda \mu \nu}+\frac{1}{2} \mathcal{T}^{\lambda \mu \nu} \mathcal{T}_{\mu \lambda \nu}-\mathcal{T}^{\lambda} \mathcal{T}_{\lambda}\right)
$$

where $\mathcal{T}_{\mu}=\mathcal{T}^{\lambda}{ }_{\lambda \mu}$, and $k=c^{3} /(16 \pi G)$. The Lagrangian density may be rewritten as

$$
L=-\frac{1}{2} k \Sigma^{\lambda \mu \nu} \mathcal{T}_{\lambda \mu \nu}
$$

where 


$$
\Sigma^{\lambda \mu \nu}=\frac{1}{4}\left(\mathcal{T}^{\lambda \mu \nu}+\mathcal{T}^{\mu \lambda \nu}-\mathcal{T}^{\nu \lambda \mu}\right)+\frac{1}{2}\left(\eta^{\lambda \nu} \mathcal{T}^{\mu}-\eta^{\lambda \mu} \mathcal{T}^{\nu}\right)
$$

Equations $(2,3,4)$ are similar to the quantities that arise in the TEGR $[7,8]$. In particular, Eq. (4) was first established in Ref. [7]. The field equations that are obtained from the variation of the action integral are given by

$$
k\left(\partial_{\mu} \Sigma^{\lambda \mu \nu}+\partial_{\mu} \Sigma^{\nu \mu \lambda}\right)=0 .
$$

The equation above is symmetric in the indices $(\lambda \nu)$, However, it can be verified by explicit calculations that the quantity $\partial_{\mu} \Sigma^{\lambda \mu \nu}$ alone is symmetric in the indices $(\lambda \nu)$, if $\Sigma^{\lambda \mu \nu}$ is written in terms of Eq. (1). For this reason, this equation can be simplified as

$$
2 k \partial_{\mu} \Sigma^{\lambda \mu \nu}=0 .
$$

We consider now a hypothetical interaction of the field $S_{\lambda \nu}$ with matter fields. Let us assume that the Lagrangian density for the matter fields $\psi_{M}$ depends also on $S_{\lambda \nu}$, i.e., $L_{M}=L_{M}\left(\psi_{M}, S_{\lambda \nu}\right)$. We define the energy-momentum tensor $T^{\lambda \nu}$ according to

$$
\frac{\delta}{\delta S_{\lambda \nu}}\left(\frac{1}{c} L_{M}\right) \equiv \frac{1}{c} T^{\lambda \nu} .
$$

where $c$ is the speed of light. We see no other interpretation for the tensor $T^{\lambda \nu}$ other than an energy-momentum type tensor. If $S^{\lambda \nu}$ is of gravitational nature, on equal footing with the tetrad fields (as discussed in the first model of Ref. [6]), then it interacts with the matter fields as the gravitational field does, possibly via the minimal coupling. ${ }^{1}$ However, it is possible (and in fact, very likely) that the spin 2 field $S_{\lambda \mu}$ interacts only with the gravitational field, according to the discussion in the last section of the article. Thus, for the time being, we assume the validity of Eq. (7). Then, in the presence of matter fields, the field equations for the spin 2 fields are assumed to be

\footnotetext{
${ }^{1}$ As discussed in Ref. [6], possible solutions for the tensor $S_{\lambda \mu}$ are given in the form $S_{\lambda \mu}=\eta_{\lambda \mu}+$ wave solution (see Eq. (14) below), and the projection of this tensor on a tetrad basis yields $S_{a b}=e_{a}{ }^{\lambda} e_{b}{ }^{\mu} S_{\lambda \mu}$, where $(a, b)$ are Lorentz indices. This latter tensor may be interpreted as an extension of the flat Minkowski (tangent space) metric tensor $\eta_{a b}=(-1,+1,+1,+1)$, which includes oscillations (fluctuations) of the background geometry. In this sense, the tensor $S_{\lambda \mu}$ might be of gravitational nature
} 


$$
\partial_{\mu} \Sigma^{\lambda \mu \nu}=-\frac{1}{2 k c} T^{\lambda \nu} .
$$

Denoting $T$ as the trace of the tensor $T^{\lambda \nu}$, it follows from the equation above that

$$
\eta_{\lambda \nu} \partial_{\mu} \Sigma^{\lambda \mu \nu}=-\frac{1}{2 k c} T
$$

With the help of this equation, and after some rearrangements, the field equation (8) is simplified as

$$
\partial_{\mu} \partial^{\mu} S^{\lambda \nu}-\partial_{\mu} \partial^{\lambda} S^{\mu \nu}-\partial_{\mu} \partial^{\nu} S^{\mu \lambda}+\partial^{\lambda} \partial^{\nu} S_{\mu}^{\mu}=-\frac{1}{k c}\left(T^{\lambda \nu}-\frac{1}{2} \eta^{\lambda \nu} T\right) .
$$

The left hand side of (10) is similar to the linearised Ricci tensor for $h_{a b}$, which is considered in the investigation of linearised gravitational waves, where $g_{a b} \simeq \eta_{a b}+h_{a b}$ (see eq. (20.10) of Ref. [12]; here we are adopting the notation of the latter reference, where $(a, b, \ldots)$ are space-time indices). Note, however, that Eq. (10) is exact, since it does not follow from any linearisation procedure.

It is straightforward do verify that the field equations (10) are invariant under the gauge transformation

$$
S^{\lambda \mu} \rightarrow \tilde{S}^{\lambda \mu}=S^{\lambda \mu}+\partial^{\lambda} V^{\mu}+\partial^{\mu} V^{\lambda},
$$

where $V^{\lambda}(x)$ is an arbitrary vector field. We may use this gauge freedom to require the tensor $S^{\lambda \mu}$ to satisfy the condition

$$
\partial_{\rho} S^{\lambda \rho}-\frac{1}{2} \partial^{\lambda} S_{\rho}^{\rho}=0 .
$$

The condition above is known as the de Donder, or Einstein, or Hilbert, or Fock gauge [12]. By requiring $S^{\lambda \mu}$ to satisfy Eq. (12), we arrive at (dropping the tilde)

$$
\partial_{\mu} \partial^{\mu} S^{\lambda \nu}=-\frac{1}{k c}\left(T^{\lambda \nu}-\frac{1}{2} \eta^{\lambda \nu} T\right) .
$$

Thus, in empty space-time we obtain the wave equation

$$
\partial_{\mu} \partial^{\mu} S^{\lambda \nu}=0
$$


In view of the anti-symmetry $\Sigma^{\lambda \mu \nu}=-\Sigma^{\lambda \nu \mu}$, we have the conservation law

$$
\partial_{\mu} T^{\lambda \mu}=0
$$

that follows from Eq. (8). Finally, note that the tensor $\mathcal{T}_{\lambda \mu \nu}$ satisfies the cyclic relation

$$
\mathcal{T}_{\lambda \mu \nu}+\mathcal{T}_{\mu \nu \lambda}+\mathcal{T}_{\nu \lambda \mu}=0
$$

a property that the third rank tensors are required do satisfy in the framework of the Fierz-Pauli theory [2].

\section{The Hamiltonian formulation}

We will develop this section in two parts. First, we carry out the Legendre transform, obtain the primary Hamiltonian and the primary constraints. In the second part we establish the Poisson brackets, analyse the constraint structure and write the time evolution equations for the field variables in the phase space of the theory. We show the equivalence of these equations with the Lagrangian field equations, and finally determine the number of the dynamical degrees of freedom of the theory.

\subsection{The Legendre transform}

In order to carry out the Legendre transform of the Lagrangian density (3), we find it more suitable to rewrite the latter in first order differential form. For this purpose, we introduce the new set of fields $\phi_{\lambda \mu \nu}$, that eventually will be eliminated by the field equations. Thus, we establish $L=L\left(S_{\lambda \mu}, \phi_{\lambda \mu \nu}\right)$.

We define the tensor $\Lambda^{\lambda \mu \nu}$ in similarity to Eq. (4),

$$
\Lambda^{\lambda \mu \nu}=\frac{1}{4}\left(\phi^{\lambda \mu \nu}+\phi^{\mu \lambda \nu}-\phi^{\nu \lambda \mu}\right)+\frac{1}{2}\left(\eta^{\lambda \nu} \phi^{\mu}-\eta^{\lambda \mu} \phi^{\nu}\right),
$$

where $\phi^{\mu}=\phi_{\lambda}^{\lambda}{ }^{\mu}$. Thus, the Lagrangian density in first order differential formulation is given by

$$
L=\frac{1}{2} k \Lambda^{\lambda \mu \nu}\left(\phi_{\lambda \mu \nu}-2 \mathcal{T}_{\lambda \mu \nu}\right) .
$$

The variation of $L$ with respect to $\phi_{\lambda \mu \nu}$ yields 


$$
\delta L=k\left[\left(\delta \Lambda^{\lambda \mu \nu}\right)\left(\phi_{\lambda \mu \nu}-\mathcal{T}_{\lambda \mu \nu}\right)\right]=0,
$$

from what we conclude that $\phi_{\lambda \mu \nu}=\mathcal{T}_{\lambda \mu \nu}$. This equation splits into two parts,

$$
\begin{aligned}
\phi_{\lambda 0 j} & =\mathcal{T}_{\lambda 0 j}, \\
\phi_{\lambda i j} & =\mathcal{T}_{\lambda i j} .
\end{aligned}
$$

The Latin indices $i, j, k \ldots$ represent space indices and run from 1 to 3 . In the $3+1$ decomposition of (18), we will use Eq. (21) to eliminate $\phi_{\lambda i j}$ from the very beginning, but the "velocity" fields $\phi_{\lambda 0 j}$ will be eliminated only at the end of the Legendre transform, after inverting the velocities in terms of the momenta.

As usual, the dot indicates time derivative, i.e., $\dot{S}_{\lambda \mu}=\partial_{0} S_{\lambda \mu}$. In Eq. (18), there is no time derivative of the field quantity $S_{00}$. The canonical momenta are obtained from the Lagrangian density through the term $\left(-2 k \Lambda^{\lambda 0 j}\right) \mathcal{T}_{\lambda 0 j}$, i.e.,

$$
\begin{aligned}
\left(-2 k \Lambda^{\lambda 0 j}\right) \dot{S}_{\lambda j} & =\left(-2 k \Lambda^{00 j}\right) \dot{S}_{0 j}+\left[-k\left(\Lambda^{i 0 j}+\Lambda^{j 0 i}\right)\right] \dot{S}_{i j} \\
& \equiv \Pi^{0 j} \dot{S}_{0 j}+\Pi^{i j} \dot{S}_{i j} .
\end{aligned}
$$

Thus, we define the momenta canonically conjugated to $S_{0 j}$ and $S_{i j}$ by

$$
\begin{gathered}
\Pi^{0 j}=-2 k \Lambda^{00 j}, \\
\Pi^{i j}=-k\left(\Lambda^{i 0 j}+\Lambda^{j 0 i}\right),
\end{gathered}
$$

respectively. The right hand side of Eq. (23) does not depend on time derivatives. It is equal to $-k \mathcal{T}^{k}{ }_{k}{ }^{j}$. As a consequence, we have the following primary constraints,

$$
\begin{aligned}
\Pi^{00} & =0, \\
\Pi^{0 j}+k \mathcal{T}_{k}^{k}{ }^{j} & =0 .
\end{aligned}
$$

The algebra that leads to the expression of the "velocities" in terms of the momenta is not intricate. By developing Eq. (24) we find 


$$
\Pi^{i j}=-\frac{k}{2}\left(\phi^{i 0 j}+\phi^{j 0 i}\right)-k \eta^{i j}\left(\eta^{k l} \phi_{k 0 l}\right) .
$$

Denoting $\Pi=\Pi^{i}{ }_{i}$, we obtain

$$
\eta_{i j} \phi^{i 0 j}=\frac{1}{2 k} \Pi
$$

Thus, Eqs. (27) and (28) lead to

$$
\phi^{i 0 i}+\phi^{j 0 i}=-\frac{2}{k}\left(\Pi^{i j}-\frac{1}{2} \eta^{i j} \Pi\right) .
$$

From the equation above we obtain

$$
\dot{S}_{i j}=\frac{1}{2}\left(\partial_{i} S_{j 0}+\partial_{j} S_{i 0}\right)+\frac{1}{k}\left(\Pi_{i j}-\frac{1}{2} \eta_{i j} \Pi\right),
$$

and

$$
\phi_{i 0 j}=\frac{1}{2} \mathcal{T}_{0 i j}+\frac{1}{k}\left(\Pi_{i j}-\frac{1}{2} \eta_{i j} \Pi\right) .
$$

With the help of the expressions above, it is not difficult to rewrite the Lagrangian density in terms of the field variables in the phase space of the theory. After a number of rearrangements and simplifications, we arrive at

$$
\begin{aligned}
L= & \Pi^{i j} \dot{S}_{i j}+\Pi^{0 j} \dot{S}_{0 j}-\frac{1}{2 k}\left(\Pi^{i j} \Pi_{i j}-\frac{1}{2} \Pi^{2}\right) \\
& -\Pi^{0 j} \partial_{j} S_{00}-\Pi^{i j} \partial_{j} S_{i 0}-\frac{3}{8} k \mathcal{T}^{0 i j} \mathcal{T}_{0 i j}-\frac{1}{2} k \Sigma^{k i j} \mathcal{T}_{k i j} .
\end{aligned}
$$

Equation (32) finally leads to the expression of the primary Hamiltonian,

$$
\begin{aligned}
H_{0}= & \frac{1}{2 k}\left(\Pi^{i j} \Pi_{i j}-\frac{1}{2} \Pi^{2}\right)+\Pi^{i j} \partial_{j} S_{i 0}+\Pi^{0 j} \partial_{j} S_{00} \\
& +\frac{3 k}{8} \mathcal{T}^{0 i j} \mathcal{T}_{0 i j}+\frac{k}{2} \Sigma^{k i j} \mathcal{T}_{k i j} .
\end{aligned}
$$

Both in (32) and in (33), the tensor $\Sigma^{k i j}$ depends only on $\mathcal{T}_{k i j}$, i.e., it does not contain the " 0 " index. 


\subsection{Poisson brackets and time evolution equations}

The Poisson brackets between any two functions in the phase space of the theory is given by

$$
\{F, G\}=\int d^{3} z\left(\frac{\delta F}{\delta S_{\mu \nu}(z)} \frac{\delta G}{\delta \Pi^{\mu \nu}(z)}-\frac{\delta F}{\delta \Pi^{\mu \nu}(z)} \frac{\delta G}{\delta S_{\mu \nu}(z)}\right) .
$$

With the help of the Poisson brackets, we will study the time evolution of the primary constraints. Considering first Eq. (25), we have

$$
\dot{\Pi}^{00}(x)=\left\{\Pi^{00}(x), \int d^{3} y H_{0}(y)\right\} .
$$

After simple calculations, we obtain

$$
\dot{\Pi}^{00}(x)=\partial_{j} \Pi^{0 j} \simeq 0
$$

Therefore, $\partial_{j} \Pi^{0 j}=0$ is a secondary constraint of the theory. The primary constraint (26) also yields a secondary constraint. After long but also simple calculations, we obtain

$$
\begin{aligned}
\frac{d}{d t}\left(\Pi^{0 i}(x)+k \mathcal{T}_{k}^{k}{ }_{k}{ }(x)\right) & =\left\{\Pi^{0 i}(x)+k \mathcal{T}^{k}{ }_{k}{ }^{i}(x), \int d^{3} y H_{0}(y)\right\} \\
& =2 \partial_{j} \Pi^{i j}(x)+k \partial_{j} \mathcal{T}^{0 j i}(x) \\
& \simeq 0 .
\end{aligned}
$$

Therefore, we have the following set of constraints,

$$
\begin{aligned}
A(x) & =\Pi^{00}(x)=0, \\
B^{i}(x) & =\Pi^{0 i}(x)+k \mathcal{T}^{k}{ }_{k}{ }^{i}(x)=0, \\
C(x) & =\partial_{j} \Pi^{0 j}(x)=0, \\
D^{j}(x) & =2 \partial_{i} \Pi^{i j}(x)+k \partial_{i} \mathcal{T}^{0 i j}(x)=0 .
\end{aligned}
$$

We recall that $A(x)$ and $B^{i}(x)$ are primary constraints, and $C(x)$ and $D^{j}(x)$ are secondary constraints. The time evolution of the constraint $C(x)$ yields $\partial_{i} \partial_{j} \Pi^{i j} \simeq 0$, which is a consequence of the constraint (41) (in view of the anti-symmetry $\mathcal{T}^{0 i j}=-\mathcal{T}^{0 j i}$ ). Note also that by taking the divergence of the constraint $B^{i}$, and considering the constraint $C(x)$, we have $\partial_{i} \mathcal{T}^{k}{ }_{k}{ }^{i}=$ 
0 . It can be verified by explicit calculations that the resulting equation, $\partial_{i} \partial_{k} S^{i k}-\partial^{k} \partial_{k} S^{i}{ }_{i}=0$, is the 00 component of the field equations (10), assuming the vanishing of the energy-momentum tensor on the right hand side of (10). Finally, the time evolution of the constraint $D^{j}(x)$ is strongly equal to zero.

It can be shown that all possible Poisson brackets between the constraints (38), (39), (40) and (41) are strongly equal to zero. In the evaluation of $\left\{B^{i}(x), D^{j}(y)\right\}$, special attention is needed in the symmetrization of the variations required by Eq. (34) (i.e., $\delta / \delta S_{i j}(z)=\delta / \delta S_{j i}(z)$ ). Thus, all constraints of the theory are first class constraints.

In principle, one would have to add the first class constraints to the primary Hamiltonian density $H_{0}(x)$, each one multiplied by a priori arbitrary Lagrange multipliers. The latter should be consistent with the field equations of the theory. However, it can be verified that the primary Hamiltonian alone, constructed out of $H_{0}(x)$ only, already yields field equations that are strictly equivalent to the Lagrangian field equations. First, it is very easy to show that Hamiltonian field equation

$$
\dot{S}_{i j}=\left\{S_{i j}(x), \int d^{3} y H_{0}(y)\right\}
$$

yields exactly Eq. (30). As for the field equation for the momenta $\Pi^{i j}=\Pi^{j i}$, it is not difficult to conclude that

$$
\begin{aligned}
\dot{\Pi}^{i j} & =\left\{\Pi^{i j}(x), \int d^{3} y H_{0}(y)\right\} \\
& =k\left(\partial_{m} \Sigma^{i m j}+\partial_{m} \Sigma^{j m i}\right) .
\end{aligned}
$$

Let us now identify $\phi_{i 0 j}=\mathcal{T}_{i 0 j}$, which follows from Eq. (20), and consider this identification in Eq. (24). Then, it is straightforward to rewrite the time derivative of the momenta canonically conjugated to $S_{i j}$ as

$$
\dot{\Pi}^{i j}=-k \partial_{0}\left(\Sigma^{i 0 j}+\Sigma^{j 0 i}\right) .
$$

Combining the expression above with the right hand side of (43), we find

$$
k \partial_{\mu}\left(\Sigma^{i \mu j}+\Sigma^{j \mu i}\right)=0,
$$

which is the $i j$ component of the field equation (8), assuming the vanishing of the energy-momentum tensor in the latter. Considering the dependence of 
the tensor $\mathcal{T}_{\lambda \mu \nu}$ on $S_{\mu \nu}$, as in Eq. (1), then it is not difficult to show that each term $\partial_{\mu} \Sigma^{i \mu j}$ and $\partial_{\mu} \Sigma^{j \mu i}$ are, separately, symmetric in the indices $i j$. Making use of Eq. (1), the field equation above is rewritten as

$$
\begin{aligned}
& k\left(\partial_{\mu} \partial^{\mu} S^{i j}-\partial^{j} \partial_{\mu} S^{i \mu}-\partial^{i} \partial_{\mu} S^{j \mu}\right. \\
& \left.+\eta^{i j} \partial_{\mu} \partial_{\lambda} S^{\lambda \mu}-\eta^{i j} \partial_{\mu} \partial^{\mu} S_{\lambda}^{\lambda}+\partial^{i} \partial^{j} S_{\lambda}^{\lambda}\right)=0 .
\end{aligned}
$$

This equation is invariant under the gauge transformation (11), exactly like Eq. (10), again assuming $T^{\lambda \mu}=0$ in the latter.

The remaining Hamiltonian field equations are

$$
\begin{aligned}
\dot{S}_{00} & =\left\{S_{i j}(x), \int d^{3} y H_{0}(y)\right\}=0, \\
\dot{S}_{0 i} & =\left\{S_{0 i}(x), \int d^{3} y H_{0}(y)\right\}=\partial_{i} S_{00}, \\
\dot{\Pi}^{0 i} & =\left\{\Pi^{0 i}(x), \int d^{3} y H_{0}(y)\right\}=\partial_{j} \Pi^{i j}+\frac{3 k}{2} \partial_{j} \mathcal{T}^{0 j i} .
\end{aligned}
$$

We may now calculate the radiating degrees of freedom of the theory. In the phase space of the theory we start with $10+10=20$ degrees of freedom, and we have 8 first class constraints at each space-time event, as given by Eqs. (38)-(41). These constraints generate the expected symmetries and remove $8+8$ degrees of freedom in the phase space. Thus, there remains 4 degrees of freedom in the phase space of the theory. In the configuration space, the counting is the same. We start with 10 degrees of freedom, and observe that the gauge symmetry (11) removes 4 degrees of freedom, either from Eq. (10) or (46). The equations

$$
\begin{aligned}
& \ddot{S}_{00}=0, \\
& \ddot{S}_{0 i}=0,
\end{aligned}
$$

that follow from (47) and (48), also remove 4 degrees of freedom (the Hamiltonian density may be rewritten such that the components $S_{00}$ and $S_{0 i}$ arise as Lagrange multipliers, but the form of $H_{0}$ is more useful for our purposes), and therefore we are left with 2 dynamical degrees of freedom in the configuration space. 


\section{The energy of the spin 2 fields}

The energy-momentum of the spin 2 fields described in this article is a relevant topic that deserves a careful analysis, which will be carried out elsewhere. Here, we will just assume that the integral of the primary Hamiltonian density $H_{0}$ yields the energy of the fields. In order to evaluate this energy, let us first recall that the left hand side of Eq. (10) is exactly equivalent to the linearised Ricci tensor for $h_{a b}$, where $g_{a b} \simeq \eta_{a b}+h_{a b}$ (in the notation of Ref. [12]), and which is considered in the investigation of linearised gravitational waves. Indeed, equation (20.10) of Ref. [12] is constructed out of $h_{a b}$, and is identical to the left hand side of (10) (which is not linearised). Thus, we may adopt the gauge condition given by Eq. (12), which is also adopted in Ref. [12]. In the latter reference, it is found the general form of the tensor $h_{a b}$ that satisfies a gauge condition similar to Eq. (12). It is given by Eq. (20.48) of Ref. [12], and reads

$$
h_{a b}=\left(\begin{array}{cccc}
h_{00} & -\frac{1}{2}\left(h_{00}+h_{11}\right) & h_{02} & h_{03} \\
-\frac{1}{2}\left(h_{00}+h_{11}\right) & h_{11} & -h_{02} & -h_{03} \\
h_{02} & -h_{02} & h_{22} & h_{23} \\
h_{03} & -h_{03} & h_{23} & -h_{22}
\end{array}\right) .
$$

Making now the identification $S_{\lambda \mu} \leftrightarrow h_{a b}$, and requiring initial conditions such that $S_{00}=S_{0 i}=0$, we obtain

$$
S_{\mu \nu}=\left(\begin{array}{cccc}
0 & 0 & 0 & 0 \\
0 & 0 & 0 & 0 \\
0 & 0 & S_{22} & S_{23} \\
0 & 0 & S_{23} & -S_{22}
\end{array}\right)
$$

Both $S_{22}$ and $S_{23}$ are taken to be functions of $(t-x)$, assuming that the fields propagate in the $x$ direction. The field equations for these components are $\partial_{\mu} \partial^{\mu} S_{22}=\partial_{\mu} \partial^{\mu} S_{23}=0$.

Since $S_{\lambda \mu}$ reduce to only $S_{22}(t-x)$ and $S_{23}(t-x)$, we find

$$
\Sigma^{i i k} \mathcal{T}_{i j k}=2\left[\left(\partial_{x} S_{22}\right)^{2}+\left(\partial_{x} S_{23}\right)^{2}\right]
$$

and

$$
\Pi^{i j} \Pi_{i j}-\frac{1}{2} \Pi^{2}=2 k^{2}\left[\left(\partial_{0} S_{22}\right)^{2}+\left(\partial_{0} S_{23}\right)^{2}\right]
$$


which eventually yields

$$
H_{0}=E=2 k\left[\left(\partial_{0} S_{22}\right)^{2}+\left(\partial_{0} S_{23}\right)^{2}\right],
$$

where $2 k=c^{3} /(8 \pi G)$. The resulting expression is very simple, and should represent the energy density of gravitons in a hypothetical quantum theory.

\section{Final comments}

We have analysed the Lagrangian and Hamiltonian formulations of a theory for spin 2 fields. The structure of the theory is motivated by the teleparallel equivalent of general relativity, and some general features of the theory remind Maxwell's theory for the electromagnetic field, as for instance the field equations (6). The conclusion is that the theory is consistent. It is clear from the Hamiltonian analysis that the theory contains two dynamical degrees of freedom in the configuration space.

One important aspect of the theory is the interaction of the spin 2 fields with ordinary matter fields. This issue is not completely settled in this article. However, we may envisage the interaction of the spin 2 fields with the gravitational field. The natural coupling of the tensor field $S_{\mu \nu}$ with the gravitational field has already been addressed in Ref. [6]. We expect that the minimal coupling between $S_{\mu \nu}$ and either the tetrad fields or the metric tensor is given by equations (28) or (35) of the latter reference, which read

$$
\nabla_{\mu}\left(e \Sigma_{\lambda}^{\mu \nu}\right)=\partial_{\mu}\left(e \Sigma_{\lambda}^{\mu \nu}\right)-e \Gamma_{\mu \lambda}^{\sigma} \Sigma_{\sigma}^{\mu \nu}=0,
$$

where $e=\sqrt{-g}, g=\operatorname{det}\left(g_{\mu \nu}\right)$, and which generalize Eq. (6). In principle, the connection $\Gamma_{\mu \lambda}^{\sigma}$ in the equation above may be either the Christoffel symbols, or the Weitzenböck connection. This equation will be investigated in the weak field approximation of the gravitational field, in which case the gravitational field acts as a source of the field equations (8) or (10). Therefore, time varying gravitational fields generate the propagation of spin 2 fields.

A new aspect of the theory discussed in this article is the possible existence of static solutions of the field equations (10), in similarity to the solutions of Gauss law in Maxwell's theory. By investigating whether these solutions exist, and whether they are physical or not, we may have clues about the nature of the possible sources for the spin 2 fields. 


\section{References}

[1] M. Fierz and W. Pauli, "On relativistic wave equations for particles of arbitrary spin in an electromagnetic field, Proc. Roy. Soc. Lond. A 173 (1939), 211-232.

[2] M. Novello and R. P. Neves, "Spin-2 field theory in curved spacetime in the Fierz representation", Class. Quantum Grav. 19, 5335 (2002).

[3] S. C. Bhargava and H. Watanabe, "The Lagrangian Formalism of the Theory of Spin 2 Fields", Nucl. Phys. B 87, 273 (1966).

[4] I. L. Buchbinder, D. M. Gitman, V. A. Krykhtin and V. D. Pershin, "Equations of Motion for Massive Spin 2 Field Coupled to Gravity", arxiv:hep-th/9910188v2.

[5] C. de Rham, G. Gabadadze and A. J. Tolley, "Resummation of Massive Gravity", Phys. Rev. Lett. 106, 231101.

[6] J. W. Maluf, "Distorted Torsion Tensor, Teleparallelism and Spin 2 Field Equations", arxiv:2006.16430.

[7] J. W. Maluf, "Hamiltonian formulation of the teleparallel description of general relativity", J. Math. Phys. 35, 335 (1994).

[8] J. W. Maluf, "The teleparallel equivalent of general relativity", Ann. Phys. (Berlin) 525, 339 (2013).

[9] S. Okubo, "Nijenhuis-Bianchi identity and BRST-like operators", J. Math. Phys. 33 (3), 895 (1992).

[10] A. Nijenhuis, Indag. Math. 13, 200 (1951).

[11] M. Nakahara, "Geometry, Topology and Physics" (IOP Publishing Ltd, 1992).

[12] Ray D'Inverno, "Introducing Einstein's Relativity" (Oxford, 2002). 